\title{
Study on the Technologies of Close Range Photogrammetry and Applications in the Manufacture of Aviation
}

\author{
Zhao Gang ${ }^{1}$, Zhang Chengyang ${ }^{1}$, Jing Xishuang ${ }^{1,}$, , Ling Xuanzhe ${ }^{1}$ and Chen \\ Siyu ${ }^{1}$ \\ ${ }^{1}$ School of Mechanical Engineering and Automation, Beihang University, Beijing 100191, China \\ a tom@buaa.edu.cn
}

Keywords: close range photogrammetry; measurement accuracy; camera calibration; reverse; manufacture of aviation

Abstract. In recent years, the technologies of close range photogrammetry have been widely used in the manufacture of aviation, so the on the technologies of close range photogrammetry and applications are studied. The taking of measurement points and the calibration of close range photogrammetry are discussed. The advantages and disadvantages of close range photogrammetry are summarized. The applications of close range photogrammetry in the manufacture of aviation are described.

\section{Introduction}

With the development of digital technology, ever increasing requirements on the quality of industrial products has been posed, which puts higher requirements on the accuracy and efficiency of measurement in the manufacturing process. For example, high accuracy, large range and high efficiency are usually required in the measurement of aircraft components in the aviation's manufacturing process. As a kind of digital measurement system, the close-range photogrammetry system can simultaneously meet the measurement requirements in the aviation manufacture. That is to say, the to-be-measured object can be measured within tens of meters and the tens of thousands of points can be measured at the same time under the premise of the measurement accuracy. Thus, in recent years, close-range photogrammetry system has been widely used in the aviation's manufacturing industry.

\section{The Close-range Photogrammetry System Components}

The close-range photogrammetry system mainly consists of the three parts of calibrated camera lens, landmark points and computer. Its working principle shown is in FIG. 1. Stick the landmark points onto the to-be-measured points of the object; use the camera with calibrated lenses to take a number of photos of the measured object from different directions to generate two-dimensional digital images. The computer uses image recognition technology to determine the location of the landmark points, and then the coordinates of the landmark points and camera pose information can be obtained based on the mathematical models of collinearity equation and space intersection [1]. The data processing procedure is shown in FIG. 2 in the working process of the close-range photogrammetry. 


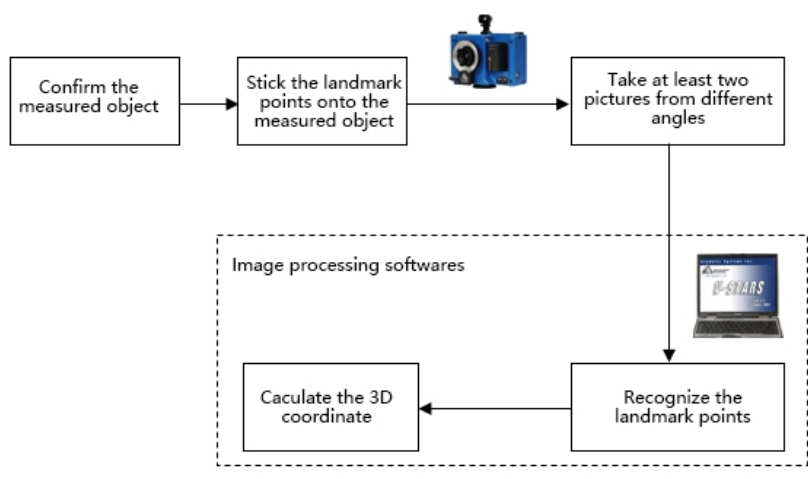

Fig.1 Principle of close range photogrammetry

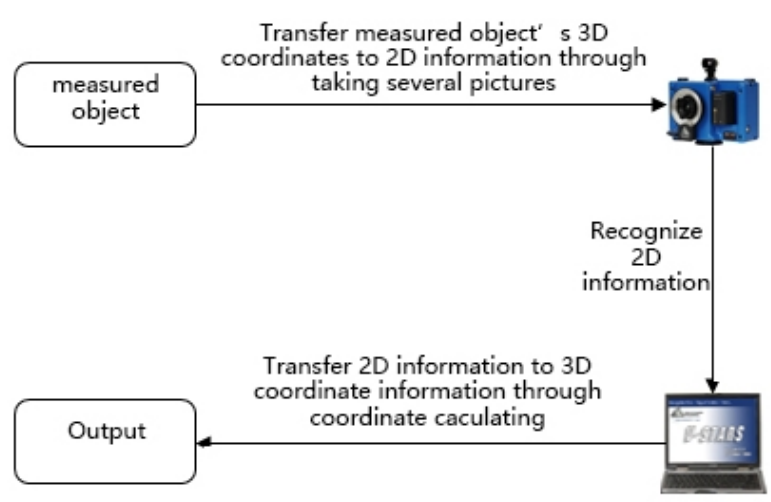

Fig.2 Data processing of close range photogrammetry

So far, various close-range photogrammetry systems have been launched by companies around the globe, such as the V-STARS 3D photogrammetry system based on high-resolution, high-precision solid-state image sensor launched by American Geodetic Survey Co Inc. (GSI); TRITOP 3D photogrammetry system launched by Germany GOM; DPA-PRO single-camera photogrammetry system launched by AICON 3D SYSTEMS GMBH company in Germany; photoelectric-type coordinate measurement system by METRONOR in Norway; METRIS K600 online photogrammetry system launched by METRIS in Belgium [1,2]. The measurement accuracy, range and features for these photogrammetry systems are listed in Table 1.

Table 1 Basic information of the existing photogrammetry systems

\begin{tabular}{|c|c|c|c|}
\hline Name of systems & $\begin{array}{c}\text { Measurement } \\
\text { accuracy }\end{array}$ & $\begin{array}{c}\text { Measurement } \\
\text { range }\end{array}$ & Features \\
\hline V-STARS & $5 \mu \mathrm{m} / \mathrm{m}$ & $50 \mathrm{~m}$ & $\begin{array}{c}\text { High precision, large measurement range, } \\
\text { wide range of applications }\end{array}$ \\
\hline TRITOP & $10 \mu \mathrm{m} / \mathrm{m}$ & $10 \mathrm{~m}$ & $\begin{array}{c}\text { Use common SLR cameras as image } \\
\text { sensors }\end{array}$ \\
\hline DPA-PRO & $15 \mu \mathrm{m} / \mathrm{m}$ & $10 \mathrm{~m}$ & $\begin{array}{c}\text { Measurement results can be imported into } \\
\text { PolyWorks, Metrolog, RapidForm and } \\
\text { Metrosoft for follow-up analysis }\end{array}$ \\
\hline METRONOR & $10 \mu \mathrm{m} / \mathrm{m}$ & $30 \mathrm{~m}$ & $\begin{array}{c}\text { Can perform the measurement of the } \\
\text { hidden points }\end{array}$ \\
\hline METRIS K600 & $12 \mu \mathrm{m} / \mathrm{m}$ & $6 \mathrm{~m}$ & Can track 100 targets simultaneously in \\
real time
\end{tabular}

The V-STARS system from GSI Company in USA has been widely used in aviation manufacturing and other fields due to its high accuracy and large measurement range. The V-STARS system has double-unit online photogrammetry models of V-STARS / DE, V-STARS / M8 and other offline photogrammetry systems of V-STARS / E5 and V-STARS / S8. The V- STARS / S8 close-range photogrammetry system is shown in FIG. 3. The main differences among the various models are caused by the different levels of hardware conFigurations of the cameras, resulting in different measurement accuracy and efficiency [3]. The accuracy of stand-alone photogrammetry system with high-conFiguration can reach up to $5 \mu \mathrm{m}+5 \mu \mathrm{m} / \mathrm{m}$, and its measurement range can reach up to $50 \mathrm{~m}$. As a domestic close-range photogrammetry system, the MPS system can be divided into two models of MPS / S stand-alone photogrammetry system and MPS / M double-unit photogrammetry system. MPS system uses high resolution LEICA or NIKON digital camera and can be used to carry out the measured in the environment of $-10^{\circ} \mathrm{C}$ to $5^{\circ} \mathrm{C}$, and its measurement range can reach up to $200 \mathrm{~m}$, but the measurement accuracy is poor compared with foreign photogrammetry system. The measurement error of domestic MPS system within $5 \mathrm{~m}$ is less than $0.05 \mathrm{~mm}$, therefore, there is still much room for improvement and upgrade in the close-range photogrammetry system. 


\section{The key technologies of close-range photogrammetry system}

The key to the photogrammetry system is to minimize the measurement error. Errors are generated in the process of information conversion, recognition and computation of the coordinates. Meanwhile, the system errors caused by the system hardware devices themselves will also affect the accuracy of the measurement results. How to control and minimize these errors is the key to the close-range photogrammetry system.

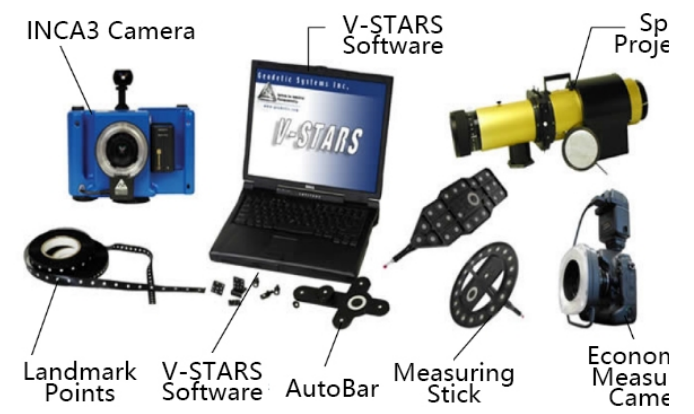

Fig.3 V-STARS close range photogrammetry

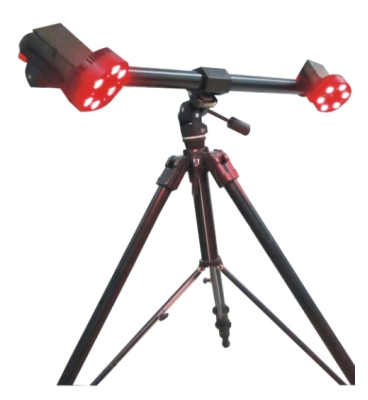

Fig.4 MPS close range photogrammetry

1) Camera calibration technology: Camera calibration technology mainly focus on the reduction of the error which is produced in the conversion of 3D coordinate information into $2 \mathrm{D}$ images when shooting photos, in fact, it is the technology that use mathematical methods to minimize the influence of camera lens distortion on the accuracy of the measurement results. Camera lens distortion is mainly caused by processing and assembly in the manufacturing process, which leads to the imperfect coaxially of lens so that the refraction phenomenon occurs when light passes through the lens, making the measurement results fail to meet the collinearity condition, namely the measured point, the center of the lens and the image point cannot meet the collinearity condition [4,5]. In order to cope with the distortion of the camera, the mathematical model is usually adopted for compensation and calibration. At present, a variety of mathematical models for camera calibration and compensation were proposed by domestic and abroad scholars, such as ten-parameter model, polynomial model, finite element model, artificial neural network [2, 6-8]. These different mathematical models were constructed to improve the accuracy of the calibrations based mainly on the camera lens distortion size and the strength of the distortion law. When performing the close-range photogrammetry with the calibrated camera, the space coordinate of the to-be-measured point and the relative pose of the camera are calculated according to the algorithm on collinearity equation and space intersection.

2) The recognition technology of measurement points: In the close-range photogrammetry technology, after photographing the to-be-measured object affixed with the landmark points, the accurate recognition of the landmark points from the digital image is a prerequisite for computation of the space coordinate of the object to be measured. The recognition technology of measurement points mainly focuses on the reduction of the errors produced in the image recognition of landmark points. The digital images photographed by the close-range photogrammetry technology are black and white, so the image processing software recognizes the landmark points by analyzing the gray value of each pixel in the image. Since the landmark points are made of reflector material whose brightness value is larger than the surrounding objects so that the positions of the landmark points in the digital image can be recognized. Various landmark recognition algorithms were proposed by scholars, domestic and abroad, such as Canny edge detector method, line scanning technique, recursive methods of filling as well as morphological analysis [9-11]. After recognizing the positions of the landmark points, we also need to compute the coordinates of the center of the landmark points. Many algorithms have been proposed for calculating the center of the landmark points so far, such as ellipse fitting method, gray weighted centroid method and template matching method [9-13] which mainly compute the coordinates of the center point by determining the edge shape and the gray value of the landmark points, 
and the ellipse fitting method is suitable for processing the image with hot pixel. The gray weighted centroid method is suitable for processing the image of the landmark points with smaller radius.

3) Automatic image matching technology: In the close-range photogrammetry, it is necessary to take a number of images of the to-be-measured objects from a number of directions, and then image processing software will automatically match the different images with the same landmark points together to calculate their spatial coordinates. To achieve automatic image matching, the encoded points should be pasted around the objects and points to be measured. The encoded points are patterned points which are designed to have numbering function and can be recognized by the image processing software. In all the digital images photographed, the encoded points with the same numbering will be automatically matched by the image processing software to find the position of the same landmark point in the different digital images. In close-range photogrammetry technology, the automatic matching of images is mainly carried out by the spatial relationship of the image point space, and the matching algorithm based on or with the polar constraint is usually used [9, 14].

4) Computation of coordinates' technology of the points to be tested: The computation of coordinates' technology of the points to be tested mainly focuses on the reduction of the errors generated in the coordinate computation process. In the close-range photogrammetry system, the most widely used coordinate computation algorithm is self-calibrating bundle adjustment method which calculates the parametric values of the to-be-measured point and the camera pose by treating pinpointed coordinates as the observed values, and using collinearity equations and adjustment method. At present, self-calibrating bundle adjustment method includes several methods [1] of pointwise elimination method and total elimination method. For different measurement environments, it is helpful to reduce the error caused by the calculation process by choosing appropriate computational algorithm.

5) System error control technology: The accuracy of measurement will be affected by camera resolution and hot pixels etc. The level of details measured in pixels captured by the camera is called the resolution of the camera. That is to say, the more effective pixels contained in the image, the greater the resolution of the camera [15]. In the close-range photogrammetry system, the relative measurement accuracy is the ratio of extracted accuracy of image point coordinates to image resolution [16]. Once the extracted accuracy of image point coordinates is given, the higher the resolution of the camera is, the higher the relative measurement accuracy. The hot pixels refer to rough part produced in the images in the processes of receiving or outputting light through camera's CCD (CMOS) sensor, and is usually caused by electronic interference. The hot pixels in the camera produce additional pixels in the image, indicating that the color which is not found in the original image will show. When recognizing the points to be measured, the image processing software will extract the center coordinates of the circular landmark point according to the gray distribution of the reflecting mark. For example, in the fitting algorithm recognizing the ellipse border of the landmark, the hot pixels will affect the gray value at the edges, thereby reducing the accuracy of the edge recognition and causing the calculation error of the center point coordinate. Therefore, in the close-range photogrammetry system, the camera with high signal-to-noise ratio is usually selected to reduce the influence of hot pixels on the measurement accuracy.

In addition, benchmark scale within the system can also affect the accuracy of the measurement results. In the close-range photogrammetry system, one or more benchmark scales are usually placed around the object to be measured and their lengths are required to be precise and known to ensure that the measured lengths of the coordinate system are very accurate in the measurement result of the photogrammetry system. Therefore, the manufacturing errors of the benchmark scale, the deformation caused by the factors of the heat and force will have an impact on the measurement accuracy. Thus, in order to reduce the error caused by the benchmark scale, a material that is not easily deformed is generally chosen in the manufacturing process of the benchmark scales, and their lengths are measured by a laser tracker with superb precision. 


\section{The features of the close-range photogrammetry technology features and its application in aviation manufacturing}

The close-range photogrammetry technology has the following features [17]:

I High measurement accuracy. In the close-range photogrammetry system with single-camera, if equipped with the higher level of configuration, the relative measurement accuracy can reach $5 \mu \mathrm{m}+5 \mu \mathrm{m} / \mathrm{m}$.

I High measurement efficiency. The system can acquire tens of thousands of data points to be measured in a short period of time.

I Stable performance. In the case of harsh measurement environment, such as vibration, high and low temperature, high and low pressure, toxic and harmful environment, high-precision measurement can still be achieved.

I Carry out the contactless measurement. In the measurement, use the spot projector of PRO-SPORT for dot distribution, to achieve contactless measurement, where the object cannot be damaged.

Compared with other industrial measurement techniques, the close-range photogrammetry technology has unique advantages in measurement accuracy, measurement range, measurement efficiency and so on. Table 2 shows the advantages and disadvantages of various industrial measurement systems for comparison.

Table 2 The advantages and disadvantages of various industrial measurement systems

\begin{tabular}{|c|c|c|}
\hline Name of system & Advantages & Disadvantages \\
\hline $\begin{array}{c}\text { The close-range } \\
\text { photogrammetry } \\
\text { system }\end{array}$ & $\begin{array}{c}\text { High accuracy, high efficiency, good } \\
\text { stability, contactless measurement, and } \\
\text { large measurement range }\end{array}$ & Low degree of automation \\
\hline $\begin{array}{c}\text { Laser tracking } \\
\text { measurement system }\end{array}$ & High accuracy & $\begin{array}{c}\text { Low efficiency in point-wise } \\
\text { measurement }\end{array}$ \\
\hline Articulated arms & Can measure the hidden point & $\begin{array}{c}\text { Small range of measurement, } \\
\text { low efficiency in point-wise } \\
\text { measurement }\end{array}$ \\
\hline iGPS & Large range of measurement, \\
high efficiency & Low accuracy (only 0.2 mm) \\
\hline
\end{tabular}

Compared to other measurement techniques, the close-range photogrammetry technology has many obvious advantages, thus it has been widely used in the aviation industry. Due to features of the high measurement accuracy and large measurement range of the close-range photogrammetry technology, the close-range photogrammetry technology can be used to measure larger objects and ensure high measurement accuracy. Therefore, the close-range photogrammetry technology is mostly applied to the measurement of geometric features of aircraft components and parts as well as the measurement in whole aircraft.

1) Measurement of geometric features of components and parts: High precision requirements for the processing and assembly of components and parts are necessary in aerospace manufacturing sector, and the geometric features of components and parts are more complicated. Performing the close-range photogrammetry of already machined or assembled parts and components can confirm whether geometric features of components and parts comply with the quality inspection requirements. FIG. 5 shows the measurement of aileron fixture of Boeing 777 by using the V-STARS close-range photogrammetry system. The coordinate information of key points on the aileron fixture was measured and the position information of the groove, contour profile of the fixture as well as the other features of the aileron fixture were tested. The close-range photogrammetry is also suitable for measuring the assembling and docking of parts and components, measuring the geometric information of the interface part of the components and parts to ensure that the two parts and components can be fully docked.

2) The whole aircraft measurement: The close-range photogrammetry technology can also be used for whole aircraft measurement. The operators use it to check the overall outer structure of the 
aircraft, the size of the important parts, and the deformation of the key points in the aircraft before and after the flight test, so as to achieve the test of the aircraft's quality. The use of close-range photogrammetry system can accurately and quickly measure the aircraft shape; it only takes about tens of minutes for the operators to complete the whole measurement of the aircraft. Compared with the traditional method, the close-range photogrammetry greatly improves the measurement efficiency, and improves the measurement accuracy to the level of $\mu \mathrm{m}$. The development process of domestic large aircraft is shown in FIG. 6, where the close-range photogrammetry system of V-STARS has been used to carry out aircraft shape measurement. Because of its high measurement efficiency with thousands of data points being measured simultaneously, the close-range photogrammetry can be used in reverse engineering measurements.

3) Reverse engineering measurements: In the field of aerospace manufacturing, reverse engineering measurements can be implemented on a machined component by using the close-range photogrammetry system to collect coordinate information of a large number of points on the surface of a part, thereby, constructing a digitalized model. By affixing a few dense landmark points to the object to be measured or using the spot projector device to cast the spots on the surface of the object to be measured, the close-range photogrammetry system can quickly and accurately obtain the spatial coordinate information of these dense points on the surface of the object to be measured to provide the data for the digitalized model. As shown in FIG. 7, the Australian Air Force conducted reverse engineering measurements for C130 Fighter.

Using the spot projector in the close-range photogrammetry system can skip the process of affixing the landmark points to the object to be measured, enabling the contactless measurement of the close-range photogrammetry. Without influencing the objects, such contactless measurement can implement the dynamic measurement on the object whose shape has been experiencing the changes. At the same time, it can still maintain the high-precision measurement in the harsh environment due to the superb stability of the close-range photogrammetry technology; moreover, it is also applied to the wind tunnel experiment.

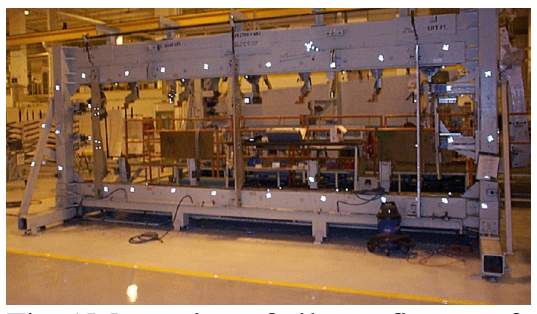

Fig.5 Measuring of aileron fixture of Boeing 777

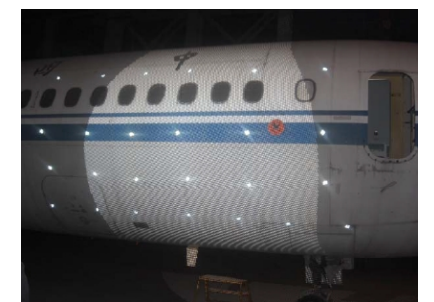

Fig.6 Measuring the appearance of large aircraft by close range photogrammetry

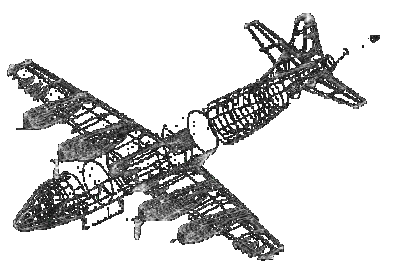

Fig.7 Reverse engineering measurements for $\mathrm{C} 130$

4) Sheet metal's deformation analysis: In the processing of aircraft parts and components, sheet metal structure often involves deformation issues, the close-range photogrammetry system with online dual-camera is used to perform dynamic measurement on shape changes of sheet metal in metal sheet processing, thereby, providing data for the force analysis [18]. At the same time, in the wind tunnel test, the close-range photogrammetry system can be used to detect the real-time performance and shape changes of the aircraft parts and components during the experiment [19].

\section{The future prospects of the close-range photogrammetry}

Since the birth of the close-range photogrammetry technology in the 30s of last century, it has been undergoing the development for several decades. In recent decades, close-range measurement technology evolved rapidly in both hardware and software algorithms and great changes have been taking place with each passing day [20]. In recent years, advanced foreign technologies have been constantly introduced into the domestic universities and enterprises and domestic close-range photogrammetry technologies have been gradually developed [21-23]. The future development of close-range photogrammetry technology in aviation manufacturing can be summarized as follows: 
(1) The application in the docking process of aircraft parts and components. The close-range photogrammetry system can be used to achieve dynamic measurement. By measuring the current coordinate information of the aircraft parts and components and comparing it with the final docking position, the photogrammetry system can be used to monitor the docking process of aircraft components and parts to ensure the docking accuracy through the calculation of the distance as well as direction along which the components and parts of the aircraft are needed to move.

(2) Application in the aircraft automatic drilling and riveting robot. The close-range photogrammetry system can be used to output the real-time coordinates of the point to be machined and guide the aircraft automatic robot to complete the drilling riveting work. The system can be used to measure the track along which the robot needs to move, thereby, guiding the robot to the specified location by automatic movement. Moreover, the position coordinates of the center point of the machining tool of a robot can be monitored so as to ensure the positioning accuracy of drilling and riveting stations of the aircraft wings.

(3) Application in the monitoring of aircraft takeoff and landing process. Take advantage of the timeliness and high efficiency of the close-range photogrammetry system to monitor the takeoff and landing process of the aircraft and analyze the performance and deformation status of the aircraft parts and components during take-off and landing processes.

\section{References}

[1] Guiping. Huang. The study and application of key technologies in the digital close-range industrial photogrammetry [D]. Tianjin University, 2005.

[2] Qiqiang Feng. Research and practice of digital industrial photogrammetry [D]. The PLA Information Engineering University, 2010.

[3] Wenhao Feng. Introduction of V-STARS industrial photogrammetry system[J]. Journal of Geomatics, 2000,04:42-47.

[4] S. Robson,M. R. Shortis. Practical Influences of Geometric and Radiometric Image Quality Provided By Different Digital Camera Systems[J]. The Photogrammetric Record,2003,16 ( 92 ) : 225-248.

[5] F Remondino, C Fraser. Digital camera calibration methods: considerations and comparisons [J]. International Archives of Photogrammetry, Remote Sensing and Spatial Information Sciences, 2006, 36(5): 266-272.

[6] Wenhao Feng, Haoliang Shang, Wenguang Hou. A digital distortion model for all kinds of imaging systems[J]. Geomatics and Information Science of Wuhan University, 2006,02:99-103.

[7] Kavzoglu T and Karsli F. Calibration of a digital sigle lens reflex(SLR) camera using artific neural networks[J]. IAPRS,2008, Vol.36(B5): 27-32.

[8] Tecklenburg W, Luhmann T, Hastedt H. Camera modelling with image-variant parameters and finite elements[C]. Heidelberg:2001: .

[9] Otepka JO, Hanley HB, Fraser CS (2002) Algorithm developments for automated off-line vision metrology[J]. Int Arch Photogramm Remote Sens 5:60-67

[10]Clarke T A. An analysis of the properties of targets used in digital close range photogrammetric measurement[J]. Videometrics III ,1994, Vol.SPIE Vol.2350: 251-262.

[11] Shortis M R, Clarke T A, and Short T. A comparison of some techniques for the subpixel location of discrete target images[J]. Videometrics III ,1994, Vol.SPIE Vol.2350: 239-250.

[12] Otepka J. Precision target mensuration in vision metrology[D]. Technische Universitaat Wien,2004.

[13] Anchini R, Beraldin J -, and Liguori C. Subpixel location of discrete target images in close-range camera calibration: a novel approach[J]. Proc. SPIE, 2007, Vol.6491(Videometrics IX). 
[14] Ariyawansa D D A P and Clarke T A. High speed correspondence for object recognition and tracking[J]. SPIE 1997, Vol.3174(Videometrics V): 70-79.

[15] Wenhao Feng. Close range photogrammetry[M]. Wuhan University Press, Wuhan, 2001.

[16] Uffenkamp V. State of the art of high precision industrial photogrammetry.[C]. In Third international workshop on accelerator alignment.Annecy, France:1993: 153-165.

[17]Qi Yong. The basic technology its application of the close-range photogrammetry[J]. Ability and Wisdom, 2013,19:190.

[18] Xiang Guo,Jin Liang,Zhenzhong Xiao,Binggang Cao. Digital image correlation for large deformation applied in Ti alloy compression and tension test[J]. Optik - International Journal for Light and Electron Optics,2014,12518:.

[19] Mark R. Shortis,James W. Seager. A practical target recognition system for close range photogrammetry[J]. Photogram Rec,2014,29147:.

[20]Lei Wang. Theoretical research and practice of digital close - range photogrammetry[D]. The PLA Information Engineering University,2002.

[21] Yueguang He, Xiumei Wang, Zhuoqiao Zeng. Digital close-range photogrammetry system and its application[J]. Mining and Metallurgical Engineering , 2001,04:1-3.

[22] Meifang Zhao, Bangxing Shen, Xiaoming Wu, Wenjun Wu, Shenghuai Wang. Aplication of rapid ambiguity-avoiding feature matching in multi-view stereo vision inspection system[J]. Journal of china institute of metrology,2003,02:9-13.

[23] Shengju Jia, Jingtao Yu. Application of Digital Close-range Photogrammetry to Inspection of Machine Parts[J]. Acta Geodaetica et Cartographica Sinica,2002,S1:61-65. 